\title{
Johannes Gebauer \\ Interpretationspraktische Stemmatik. Philologische Methoden in der \\ Interpretationsforschung am Beispiel annotierter Notenausgaben \\ von Rodes 24 Capricen und Beethovens Violinkonzert
}

Die Interpretationsforschung als vergleichsweise junge Disziplin der Musikwissenschaft erfordert oft neue Herangehensweisen, allein schon, da sie in vielen Fällen Quellen konsultiert, die bisher entweder überhaupt nicht für die Musikwissenschaft herangezogen oder unter völlig anderen Gesichtspunkten ausgewertet wurden. In einigen Fällen lassen sich dennoch bereits etablierte Untersuchungsmethoden für die Interpretationsforschung nutzen, um sie auf solche bisher weitgehend unerschlossene Quellen anzuwenden. Ein Beispiel sind die sogenannten praktischen Ausgaben des I9. und frühen 2o. Jahrhunderts, die in traditionellen musikwissenschaftlichen Diskursen überwiegend als wenig wertvoll für die musikwissenschaftliche Forschung und im besten Falle als Zeugnis eines veralteten, heute nicht mehr zeitgemäßen Umgangs mit einem `Originaltext< gesehen wurden. ${ }^{\mathrm{I}}$

Durch die zunehmende Ausweitung einer historisch informierten Aufführungspraxis auf das I9. Jahrhundert erfahren diese Quellen eine Neubewertung. Vor allem für die Beantwortung aufführungspraktischer Fragen etwa zum Fingersatz oder der Stricheinrichtung der Streicher werden praktische Ausgaben und handschriftlich bezeichnete Notenquellen herangezogen. ${ }^{2}$ Auch einige Einzeluntersuchungen konnten zeigen, 3 dass praktische Ausgaben für die aufführungs- und interpretationspraktische Forschung ergiebige Quellen darstellen, deren Potenzial erst nach und nach erschlossen wird. Neben praxisorientierten Herangehensweisen werden auch vergleichende Methoden genutzt, um Eingriffe eines Herausgebers erkennen und einordnen zu können und sowohl den Umgang mit dem Originaltext als auch die instrumentalspezifische Realisierung dessel-

1 Das SNF-Forschungsprojekt » Die Idee des Componisten ins Leben zu rufen - Instruktive Notenausgaben als Basis für historisch orientierte Interpretationsforschung im Repertoire des I9. Jahrhunderts« (20II-20I6) an der Hochschule der Künste Bern нкв konzentrierte sich insbesondere auf die sogenannten instruktiven Notenausgaben, die sich an professionelle und angehende Musiker richten; vgl. www.hkb-interpretation.ch/index.php?id=I40 (I3. Juni 20I9).

2 Vgl. beispielsweise das von Clive Brown geleitete Forschungsprojekt an der University of Leeds, »Collection of Historical Annotated String Editions (CHASE). I $9^{\text {th }}$ and Early 2oth-Century Annotated Editions of String Music. Bibliographical Problems, Editorial Content and Implications for Performance Practice«, http://chase.leeds.ac.uk (2. Februar 20I9).

3 Vgl. beispielsweise Vasiliki Papadopoulou: Zur Editions- und Aufführungsgeschichte von J. S. Bachs Sonaten und Partiten für Violine solo in der Zeit von I802 bis I940, unv. Diss. Universität für Musik und Darstellende Kunst, Wien 2015. 
ben zu beschreiben. Es wird dabei durchaus erkannt, dass die verschiedenen Herausgeber oft in Traditionslinien zueinander stehen und sich dies möglicherweise in der praktischen Einrichtung zeigt, etwa wenn Details derselben ähnlich oder identisch sind. Dabei stand allerdings in der Regel eine inhaltliche Bewertung im Vordergrund, bei welcher die Bedeutung der Einrichtung für den Interpreten oder die Praxis untersucht wurde.

In der Editionsphilologie, sowohl in der Literatur- als auch in der Musikwissenschaft, wird die sogenannte textkritische Methode angewendet, um aus mehreren Überlieferungen eines Textes einen 〉Original- $<$ beziehungsweise >Urtext $<$ zu rekonstruieren. Dafür werden die Quellen in ihren Details verglichen; im zweiten Schritt wird anhand der Übereinstimmungen und Lesarten ein Stemma abgeleitet, welches die Abhängigkeiten einzelner Quellen und Fassungen offenlegen soll. Dabei wird auf die inhaltliche Bewertung zunächst verzichtet; vielmehr wird versucht, eine Chronologie der Entstehung der Quellen sowie deren Beziehungen an philologischen Details zu erkennen. In vielen Fällen lässt sich so relativ schlüssig rekonstruieren, welche dieser Lesarten aus den oft verlorenen Urquellen stammen und was in späteren Überlieferungen nachträglich hinzugefügt oder verändert wurde.

Obwohl es bei der Arbeit mit praktischen Ausgaben in der Regel nicht um die Rekonstruktion eines >Originaltextes ‘ geht, lässt sich die etablierte textkritische Methode der Editionsphilologie nutzen, um Abhängigkeiten einzelner Ausgaben eines Werkes zu erkennen und darzustellen. Durch die Abkoppelung von inhaltlichen Fragen lassen sich - ähnlich wie in der Philologie - textliche Abhängigkeiten objektivieren und so Bezüge oder auch Abgrenzungen sichtbar machen, die durch eine inhaltliche Bewertung allein nicht ausreichend belegbar wären. Es soll hier anhand von einigen Beispielen gezeigt werden, welches Potenzial diese Methode für die Interpretationsforschung bietet.

I. Ein geeignetes Untersuchungsobjekt für die Erprobung dieser Methode findet sich in den zahlreichen Ausgaben der 24 Capricen für Violine allein von Pierre Rode (I774-I830). Dieses Werk kann als eine Art Kompendium der zu Beginn des I9. Jahrhunderts modernen französischen Interpretationspraxis der Viotti-Schule verstanden werden, als deren berühmtester Vertreter und Prototyp eines »ächt classischen Violinstils« Rode um I8Io angesehen wurde. ${ }^{4}$ Die Capricen selbst sind keineswegs nur als Etüden zur Einübung spezifischer Techniken zu sehen; vielmehr sind diese Techniken in ausgesprochen kunstvoll gearbeiteten Solostücken gefasst, sodass nicht nur die Technik, sondern

4 Vgl. Julius Rühlmann: Die Kunst des Violinspiels. Eine historische Studie [Teil 7], in: AmZ, NF 3 (I865), Sp. 68I-687, hier Sp. 684. 
auch die Vortragsart beziehungsweise die Ausdruckspalette dieser Schule vermittelt wird. 5

Dieses Werk eignet sich vor allem wegen der zahlreichen Neuausgaben im I9. und frühen 20. Jahrhundert für eine vergleichende Untersuchung. Begonnen bei den Originalausgaben konnten mehr als zwanzig Ausgaben untersucht werden, die einen Zeitraum von annähernd einhundert Jahren von I8I8 bis I9I5 abdecken; unter den Herausgebern finden sich zahlreiche namhafte Geiger des I9. und frühen 20. Jahrhunderts. Als letzte untersuchte Ausgabe wurde jene von Lucien Capet gewählt; einerseits handelt es sich dabei um die letzte Ausgabe vor Ende des Ersten Weltkriegs, der als wichtiger Einschnitt auch für die Interpretationsgeschichte gesehen werden muss, andererseits ist gerade Capet durch seine pädagogische Tätigkeit ein wichtiges Bindeglied vom I9. zum 20. Jahrhundert, da er einige der schulbildenden Pädagogen des 20. Jahrhunderts - wie Jascha Brodzky und Ivan Galamian - ausgebildet hat.

Die zwei Erstausgaben des Werks erschienen offenbar zeitgleich I8ı8 bei J. Frey in Paris und C.F. Peters in Leipzig, ${ }^{6}$ beide Ausgaben verweisen auf dem Titelblatt auf die jeweils andere. Es handelt sich dabei nicht um eine Druckplattenübernahme, sondern um voneinander unabhängige Notenstiche. Die Erstausgaben sind, neben der ausführlichen dynamischen Bezeichnung, bereits weitgehend lückenlos mit Fingersätzen und Strichbezeichnungen versehen und vollständig metronomisiert, was spätere Eingriffe in Rodes Originalbezeichnungen umso relevanter erscheinen lässt. Der Notentext der beiden Originalausgaben ist überwiegend identisch, weist jedoch einige Unterschiede in Details auf, die für die weitere Untersuchung wesentlich sind. In beiden Ausgaben finden sich gelegentliche, voneinander unabhängige Fehler; auch zeigen sich einige Abweichungen zwischen den Editionen, bei denen nicht mit Gewissheit gesagt werden kann, welche Version die >richtigere ist. So sind bei den Akzentbezeichnungen gelegentliche Unterschiede zu finden, beispielsweise nutzt Peters in der zweiten Caprice in den Takten 25 bis 28 ein $\boldsymbol{f} \boldsymbol{p}$, während Frey hier das einfache >-Betonungszeichen verwendet.7 In der elften Caprice weicht in Takt 49 der Notentext der Ausgaben voneinander ab:

5 Die Untersuchung sämtlicher Ausgaben der 24 Capricen von Pierre Rode von I8I8 bis I9I5 erfolgte im Rahmen des Forschungsclusters zur sNF-Förderungsprofessur von Kai Köpp an der Hochschule der Künste Bern.

6 Pierre Rode: ${ }_{24}$ Caprices en forme d'Etudes pour le Violon, Dans les 24 Tons de la Gamme. Dédiés à Monseigneur le Prince Chimay, Paris: J. Frey [1818]; Pierre Rode: Vingt-Quatre Caprices. En forme d'Etudes pour le violon seul dans les Vingt-Quatre tons de la Gamme composés et dédiés à Monseigneur le Prince Chimay, Leipzig: C. F. Peters [1818].

7 Drucktechnische Gründe hierfür sind auszuschließen, da sich bei Peters an anderen Stellen ebenfalls das Zeichen > findet. 

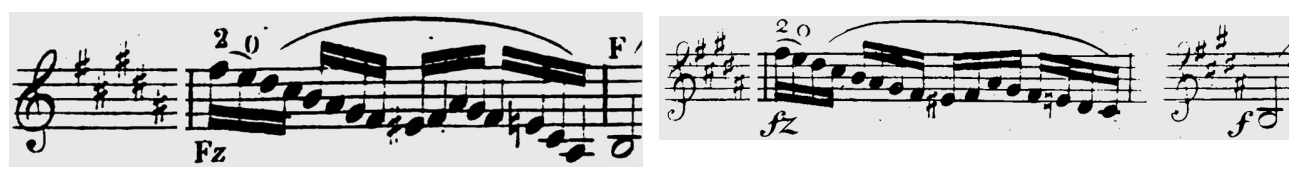

A в в IL D U N 1 Caprice Nr. 11, Takt 49, in den Ausgaben Frey 1818 (links) und Peters 1818 (rechts)

In beiden Originalausgaben fehlen gelegentlich Fingersätze, die in der jeweils anderen Ausgabe vorhanden sind. All diese Unterschiede sind für die spätere Einordung von Abhängigkeiten und die daraus resultierende Stemmatik von Bedeutung. Völlig überraschend ist die Erkenntnis, dass es offenbar zwei Versionen der Peters-Ausgabe gibt, die sich nur in einem sehr geringfügigen, aber für die weitere Editionsgeschichte ungemein wichtigen Detail unterscheiden. Während eine dieser Varianten im vierten Takt der vierten Caprice den identischen Notentext wie die Pariser Ausgabe enthält, weicht die andere hier ab; die Kadenz endet hier statt auf einem einfachen $h$ " auf einer Terz mit einem zusätzlichen $\mathrm{g}^{\prime \prime} \mathrm{P}^{2}$
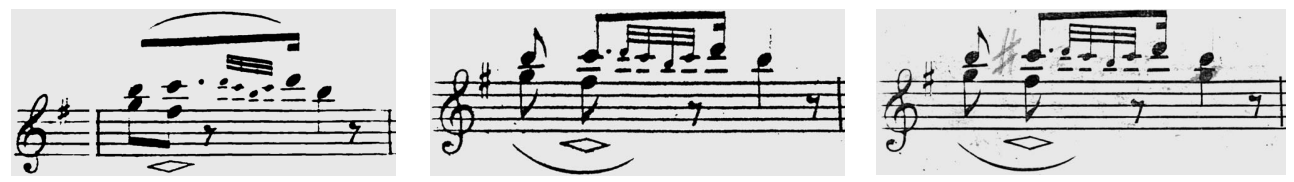

A в B ILdung 2 Caprice Nr. 4, Takt 4, in den Ausgaben Frey 1818 (links),

Peters 1818a (Mitte) und Peters 1818b (rechts)

Es ist völlig unklar, ob es sich bei einer der beiden Versionen der Peters-Ausgabe um eine nachträgliche Korrektur der Druckplatten handelt, die ansonsten völlig identisch zu sein scheinen und auch dieselbe Plattennummer haben. Da beide Lesarten - hier als Lesart a und Lesart b bezeichnet - in späteren (eindeutig auf den Peters-Ausgaben basierenden) Neuausgaben auftauchen, müssen von beiden Versionen Exemplare im Umlauf gewesen sein. Lesart b findet sich außer in Exemplaren der Originalausgabe ausschließlich in drei Neuausgaben von in Wien ansässigen Geigern, was zumindest die Vermutung erlaubt, dass diese Variante der Leipziger Ausgabe vor allem in Wien verbreitet war. Eine Chronologie lässt sich aber nicht herleiten - es ist nicht feststellbar, ob Variante b eine Revision von a darstellt oder umgekehrt.

I853 gab Ferdinand David eine Neuausgabe der Capricen bei Peters heraus, welche die Originalausgabe im selben Verlag ersetzte. ${ }^{9}$ Für seine Revision zog er sowohl die

8 Das verwendete Exemplar befindet sich in der Bibliothek der Universität der Künste Berlin unter der Signatur RA 9249. Die hier sichtbare nachträgliche Korrektur mit Bleistift erfolgte nach einer späteren Ausgabe, erkennbar am hinzugefügten \# vor c'".

9 Pierre Rode: Vingt-Quatre Caprices. En forme d'Etudes pour le violon seul dans les Vingt-Quatre tons de la Gamme composés et dédiés à Monseigneur le Prince Chimap. Nouvelle Edition, adoptée au Conser- 
Leipziger als auch die Pariser Erstausgabe heran, wie sich aus einigen Lesarten ergibt. So finden sich beispielsweise bei David einerseits in der zweiten Caprice die $\boldsymbol{f} \boldsymbol{p}$ - statt der bei Frey gedruckten >-Zeichen, andererseits folgt David in Takt 49 der elften Caprice (Abbildung I) der französischen Ausgabe. Offensichtliche Fehler korrigiert David nach der jeweils anderen Ausgabe, er ergänzt auch die gelegentlich in einer Version fehlenden Fingersätze. Darüber hinaus fügt er an einigen Stellen weitere Fingersatzziffern hinzu, wenn die Originalausgabe unklar bleibt.

Ein für den weiteren Verlauf dieser Untersuchung wesentlicher Eingriff Davids findet sich im schon erwähnten Takt 4 der vierten Caprice. David lag die Variante a der Leipziger Ausgabe vor, die er aber offenbar für unbefriedigend oder fehlerhaft hielt. Statt jedoch analog zu Variante b die Unterterz g" zu ergänzen - was im Grunde unvermeidlich gewesen wäre, hätte ihm die Variante b ebenfalls vorgelegen - ergänzte er stattdessen ein \# vor c'", was diese Kadenz nach h-Moll auflöst statt nach G-Dur:

A b B ILdung 3 Caprice Nr. 4, Takt 4 (David 1853)

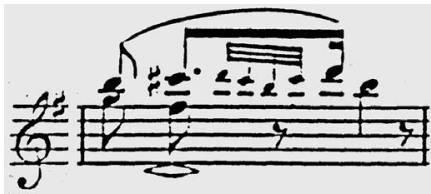

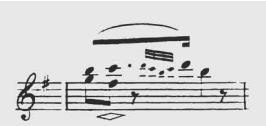

Frey 1818
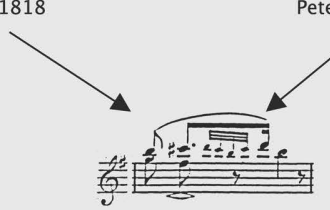

David 1853

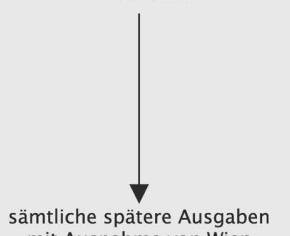

mit Ausnahme von Wien

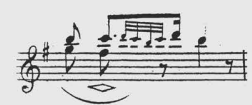

Peters $1818 \mathrm{a}$
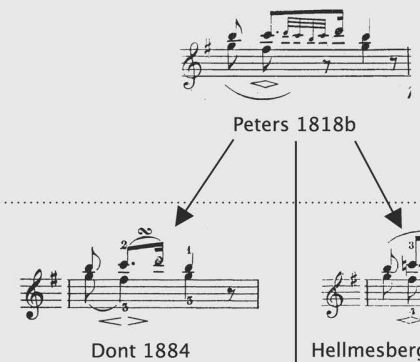

Dont 1884

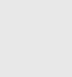

Hellmesberger sen. 1885
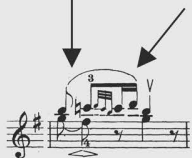

Hellmesberger jun. 1901

Ausgaben von Wiener Geigern

A в B ILd U N 4 Stemma der Vererbung, Lesarten Caprice Nr. 4, Takt 4

Anhand dieses Eingriffs lässt sich der enorm große Einfluss von Davids Ausgabe nachweisen, denn Davids Lesart, die von allen früheren Quellen abweicht, findet sich in der Folge in sämtlichen späteren Editionen - mit Ausnahme der drei Wiener Ausgaben, welche die Variante b überliefern.

vatoire de Leipzig et soigneusement revue et corrigée, hg. von Ferdinand David, Leipzig: C. F. Peters [I853]. 
Sogar Ausgaben, die ansonsten nicht von Davids Ausgabe abhängig zu sein scheinen, wie etwa jene von Lambert Massart von ı89o, überliefern das hinzugefügte Vorzeichen - offenbar hatte sich zu diesem Zeitpunkt diese Lesart bereits durchgesetzt.

Allein die Analyse der Lesarten und Vererbung von Einzelheiten der Einrichtung noch bevor jedoch eine inhaltliche Bewertung erfolgt - ergibt ein klares Stemma, welches die Beziehungen zwischen den einzelnen Ausgaben offenlegt. Der große Einfluss von Ferdinand Davids Revisionsausgabe ist im Stemma überdeutlich sichtbar, darüber hinaus ergeben sich weitere Querverbindungen, die zunächst nicht zu erwarten waren.

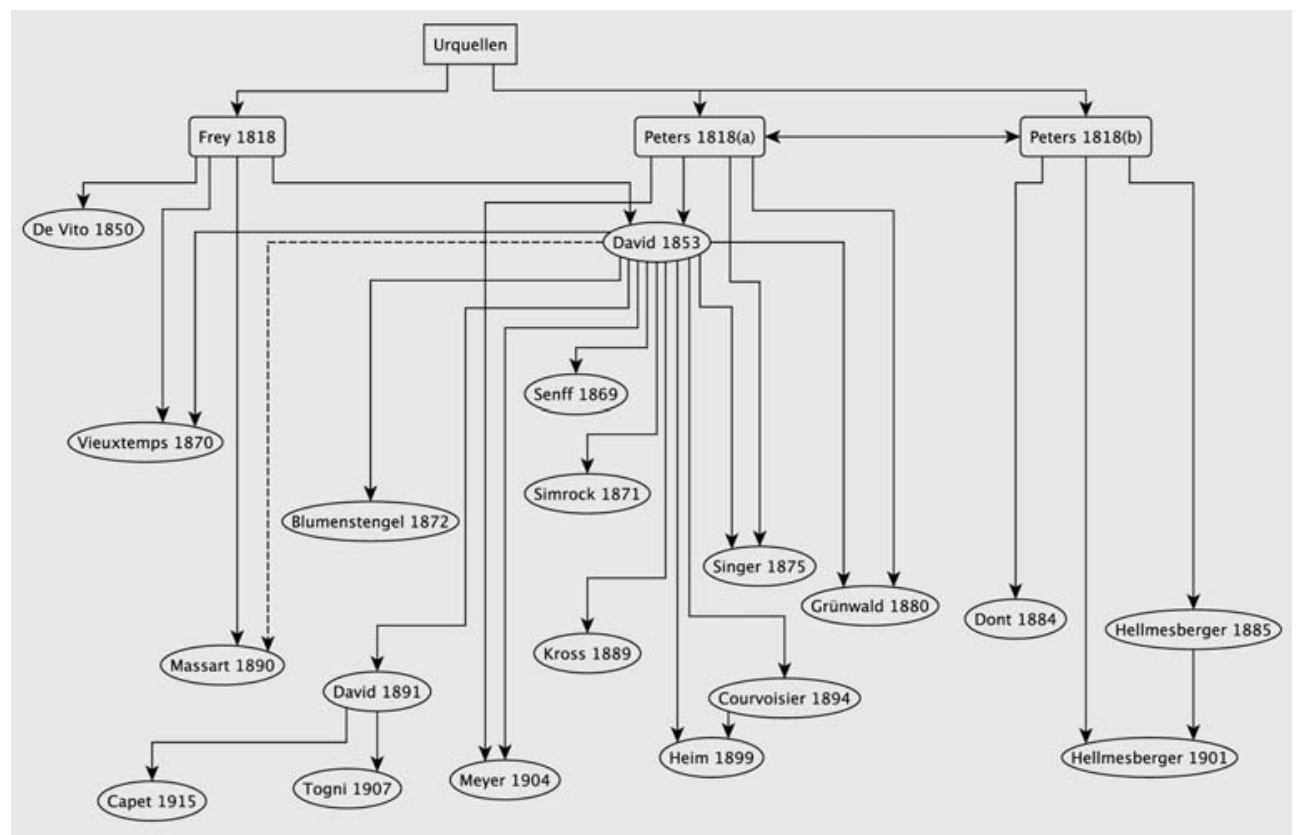

A в B ILDUNG 5 Pierre Rode: 24 Caprices, Stemma der Ausgaben von 1818 bis 1915

So hat sich Henri Vieuxtemps auf die Pariser Originalausgabe gestützt - erkennbar ist dies beispielsweise an der Verwendung des >-Zeichens in der zweiten Caprice in Takt 25 bis 28 -, dabei aber Davids Ausgabe ebenfalls herangezogen und in vielen Fällen Davids Fingersatzergänzungen übernommen. Sein belgischer Landsmann Lambert Massart verzichtet dagegen auf diese Ergänzungen, übernimmt aber ebenfalls das von David hinzugefügte und auch von Vieuxtemps übernommene Vorzeichen im vierten Takt der vierten Caprice. Lucien Capet dagegen verwendet als Quelle für seine ausführlich kommentierte instruktive Ausgabe ${ }^{\text {IO }}$ eine Revisionsausgabe von Davids Edition von 
I89I ${ }^{\text {II }}$ erkennbar an der Übernahme sämtlicher Modernisierungen dieser Ausgabe, wie beispielsweise der portato-Notierung mit Strichen statt mit Punkten:

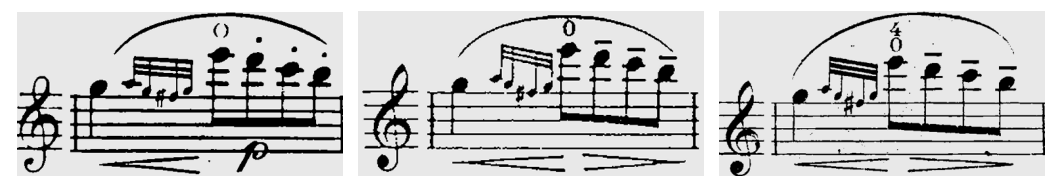

A в B IL DUNG 6 Caprice Nr. 1, Takt 3: Peters 1818 (links),

David 1891 (Mitte), Capet 1915 (rechts)

Im Stemma fällt vor allem die klare Abgrenzung der drei Ausgaben auf, die von in Wien ansässigen Geigern stammen und die sich aus der Quellenwahl und der Unabhängigkeit von anderen Herausgebern ergibt. Die weitere Untersuchung offenbart, dass diese Editionen nicht nur hierin, sondern auch in vielen inhaltlichen Details der Stricheinrichtung klar von anderen, nicht zuletzt von Rodes Originalbezeichnungen abweichen. Was sich bereits in der textkritischen Untersuchung gezeigt hat, wird später in der inhaltlichen Untersuchung überdeutlich. Die Wiener Geiger verfolgen völlig andere Stricheinrichtungsstrategien, als es von Rode vorgesehen war. Charakteristische französische Stricharten werden sowohl von Jakob Dont als auch von Josef Hellmesberger senior und dessen Sohn Josef Hellmesberger junior in vielen Fällen in sgefälligere b beziehungsweise konventionellere Stricharten abgeändert. Allen drei Wiener Ausgaben gemeinsam ist ein offensichtliches Unverständnis gegenüber klassisch-französischen Stricharten. Vor allem im Falle von Jacob Dont ist dies umso bemerkenswerter, da er als Schüler von Joseph Böhm bisher als >Enkelschüler $<$ von Pierre Rode galt. ${ }^{\text {I2 }}$

Eine so deutliche Abgrenzung der Wiener Ausgaben sowohl durch den textkritischen als auch durch den inhaltlichen Befund war zunächst nicht zu erwarten, sie deckt sich aber mit neuen Erkenntnissen zur Verwendung von Wiener Bogenmodellen und der zugehörigen Spielpraxis. ${ }^{\text {I3 }}$ Überraschend ist vor allem, dass sich die starke Abgren-

11 Pierre Rode: 24 Caprices en forme d'Etudes pour Violon seul dans les 24 Tons de la Gamme, hg. von Ferdinand David, Leipzig: C. F. Peters [189r].

12 Neuere Untersuchungen konnten allerdings belegen, dass Joseph Böhms Unterricht bei Pierre Rode nur wenige Lektionen umfasste und vermutlich viel weniger prägend war, als bisher angenommen wurde; vgl. hierzu das Kapitel »Exkurs: Joseph Böhm, ein Schüler von Pierre Rode?« in Johannes Gebauer: Der »Klassikervortrag«. Joseph Joachim und die Interpretationspraxis des I9. Jahrhunderts, unv. Diss. Universität Bern, Bern 2017.

13 Im Rahmen des Forschungsprojektes »Ein Bogen für Beethoven « an der Hochschule der Künste Bern konnte Kai Köpp unterschiedliche »repertoirespezifische Spieleigenschaften« an Wiener beziehungsweise Pariser Bögen nachweisen; vgl. www.hkb-interpretation.ch/index.php?id=I32 (4. Juni 20I7) sowie Kai Köpp: German Bows. From »Cramer Bow« to »Biedermeier Bow«, in: L'Archet Révolutionnaire Tome II [Katalog London 2015], hg. von Jérôme Akoka, Paris 2015, S. 9-I2. 
zung einer traditionellen Wiener Violinpraxis offenbar bis in das späte I9. Jahrhundert (und darüber hinaus) in den Notenquellen nachweisen lässt. Bei genauerer Betrachtung ergeben sich hier für die Geschichte des Instrumentalspiels durchaus bemerkenswerte Parallelen, etwa im Klavierbau (französische beziehungsweise Wiener Mechanik). ${ }^{14}$

Es zeigt sich, dass eine Untersuchung von praktischen Ausgaben nach editionsphilologischen Prinzipien sinnvoll ist, um Querverbindungen und Traditionslinien zwischen Herausgebern zu erkennen und offenzulegen. Das resultierende Stemma - das auf verschiedene Art und Weise ausgearbeitet und, wie im Beispiel, um eine `topografische ${ }^{\mathrm{I}} \textrm{ }$ Komponente erweitert werden kann, indem Cluster nach bekannten oder angenommenen >Schulen<, Traditionen oder auch Ausbildungs- und Aufführungsorten gebildet werden - erlaubt eine Sichtbarmachung von klaren Abgrenzungen oder Identifikationen, sei es durch die Vorlagenwahl oder durch die Übernahme der inhaltlichen Ergänzungen oder Änderungen. ${ }^{16}$

II. Wie eine solche textkritische Untersuchung für eine spezifisch interpretationsgeschichtliche Fragestellung genutzt werden kann, soll hier am Beispiel des Violinkonzertes op.6I von Ludwig van Beethoven gezeigt werden. Die Interpretation dieses Werkes wurde in der zweiten Hälfte des I9. Jahrhunderts von Joseph Joachim so stark dominiert, dass Eugène Ysaÿe Joachim als »Teil des Konzertes« bezeichnete. ${ }^{17}$ Die Forschung ging dabei bisher überwiegend davon aus, dass sich die von Joachim vertretene Auffassung

14 Auch die sogenannte Wiener Mechanik wurde von Wiener Klavierbauern bis in das frühe 2o. Jahrhundert angeboten und gebaut, so konnten noch bis Anfang des 20. Jahrhunderts Bösendorfer-Flügel wahlweise mit Wiener oder >moderner Mechanik bestellt werden. Vgl. auch Kai Köpp: French or German Bows for Beethoven. A Political Choice, London 2015, https://tarisio.com/ archet-revolutionnaire/videos/ (23. August 20I9).

15 Kai Köpp bezeichnet diese Methode als `topografische Stemmatik«, weil sie neben dem Notentext vor allem die auf die Interpretationspraxis bezogenen Textanteile in stemmatische Beziehung setzt und dadurch bislang unbekannte Zusammenhänge der Interpretationsgeschichte offenlegen kann, wie die vorliegenden Beispiele zeigen. Der Begriff ‘topografisch Hinsicht bezeichnend: Einerseits nimmt er Bezug auf interpretationspraktische >Topoi<, deren Standardisierung allein dadurch angedeutet ist, dass eine symbolische Chiffrierung vorliegt. Andererseits werden Bezüge zwischen diesen >Topoiく hergestellt, die mit einer Landschaft vergleichbare Profile und Konturen der Interpretationsgeschichte erkennen lassen, zumal tatsächlich regionale und nationale Unterschiede sichtbar werden.

16 Eine Veröffentlichung der Gesamtuntersuchung sämtlicher Ausgaben der 24 Capricen von Pierre Rode von I8I8 bis I9I5 ist in Vorbereitung.

17 »Il y a quarante ans que le maître hongrois joua cette œuvre, peu remarquée avant lui, et il la joua si bien que depuis lors, il semble inséparable de l'œuvre.« Vgl. Antoine Ysaÿe: Eugène Ysaÿe. Sa vie - son œuvre - son influence d'après les documents recueillis par son fils, Brüssel I947, S.387; vgl. auch Robin Stowell: Beethoven. Violin Concerto, Cambridge u.a. I998, S. 36. 
oder zumindest die Einrichtung mit Strichbezeichnungen und Fingersätzen an einer I844 bereits bestehenden Leipziger Aufführungstradition, vertreten durch Ferdinand David als maßgeblichem Exponenten, orientierte und diese im Wesentlichen übernahm.

Es stellt sich dabei, nicht zuletzt ausgelöst durch einige Bemerkungen von Joachims Assistenten Andreas Moser, die Frage, ob Joachim eine frühere Wiener Aufführungstradition bekannt war, die er - teilweise - mit nach Leipzig >importierte ‘, oder ob Joachim vor allem von einer bereits existierenden Leipziger Interpretation des Werks beeinflusst war, die er beispielsweise vom Spohr-Schüler Ferdinand David übernahm. Joachims eigene Ausgabe, die erst 1905 im Rahmen der Joachim/Moser-Violinschule ${ }^{\mathrm{I} 8}$ und damit erst nach Joachims letzter Aufführung des Werks, entstanden ist, kann hierzu allein keine Antwort liefern - sie stellt zwar eine wichtige Quelle zu Joachims Interpretation des Werks dar, lässt aber eine Reihe von Fragen unbeantwortet. Auch ein Exemplar der Solostimme aus Joachims Nachlass, welches sich mit einiger Sicherheit als Joachims Studierexemplar identifizieren lässt, das er I843/44 in Leipzig verwendet haben könnte, hilft hier nicht weiter, da sich darin keinerlei aufführungs- oder interpretationspraktische Zusätze wie beispielsweise Strich- oder Fingersatzbezeichnungen finden. ${ }^{\text {I9 }}$

Weitere Hinweise verspricht eine instruktiv kommentierte Ausgabe des JoachimSchülers Heinrich Dessauer, die dieser I897 mit dem Titelzusatz »Mit Bezeichnungen und Winken unter besonderer Berücksichtigung der Auffassung von Joseph Joachim für den Vortrag versehen « herausgab. Im Vergleich mit Joachims eigener Ausgabe lassen sich eine Reihe von Übereinstimmungen feststellen, die den Schluss nahelegen, dass Dessauer das Konzert während seiner Studienzeit mit Joachim erarbeitet hat und Joachims Auffassung des Werks kannte - von Joachim autorisiert war diese Ausgabe aber sicherlich nicht. Es fallen in dieser Ausgabe dagegen einige wichtige Abweichungen auf, die einer Erklärung bedürfen. Dazu lassen sich weitere Ausgaben von Joachims wie auch von Ferdinand Davids Schülern heranziehen, um eine weitere Differenzierung zu versuchen. Es soll dabei erneut zunächst einer textkritischen Herangehensweise der Vorzug gegeben werden, um - unter Berücksichtigung weiterer Quellen und Überlieferungen weitere Hinweise auf Joachims frühere Auffassung des Werks und deren Abhängigkeit von einer Wiener oder einer eventuell bereits bestehenden Leipziger Aufführungstradition zu suchen.

18 Joseph Joachim/Andreas Moser: Violinschule in 3 Bänden, Berlin u. a. I905, Bd.3, S. I85-208.

19 Bibliothek der Universität der Künste Berlin, D-Bhm, RA 46r2. Die Art der Eintragungen lässt darauf schließen, dass Joachim diese bereits beim Studium des Werkes (I844 oder früher) eingetragen hat, um sich auf das Spiel mit dem Orchester vorzubereiten. So sind beispielsweise die Paukenschläge in Takt ıo notiert - kein bereits konzerterfahrener Solist hätte dafür eine Notwendigkeit. Es finden sich in dieser Stimme sonst keine praktischen Eintragungen wie Fingersätze oder Strichbezeichnungen. 
Takt 3I4 im ersten Satz bietet sich für eine solche Untersuchung an. Die beiden Erstausgaben der Violinstimme geben in diesem Takt übereinstimmend einen ganztaktigen Bindebogen an. Ferdinand David behält diesen in seinem Gebrauchsexemplar bei und überträgt ihn auch in die von ihm eingerichtete Neuausgabe von $1865:^{20}$

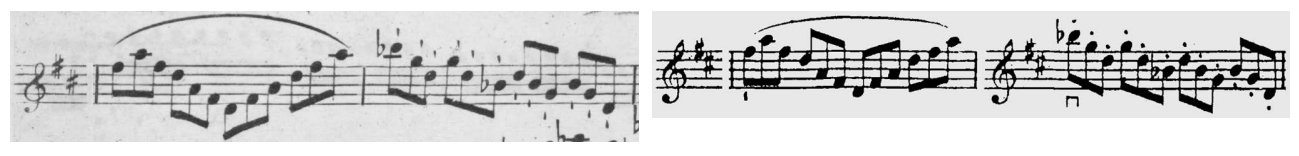

A в B I L D U N 7 Beethoven: Violinkonzert, 1. Satz, Takt $314 \mathrm{f}$.

Ferdinand Davids Gebrauchsexemplar (links) und David 1865 (rechts)

Dieser Text findet sich auch in dem offenbar von Joachim I844 genutzten Studierexemplar der englischen Erstausgabe bei Clementi, in dem sich jedoch keinerlei handschriftliche Strichbezeichnungen finden:

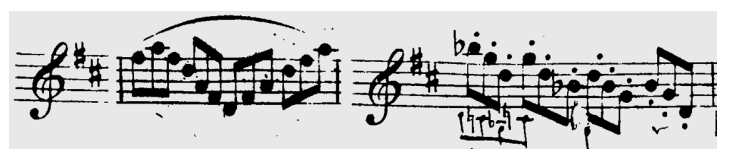

Aв в ILDUNG 8 Beethoven: Violinkonzert, 1. Satz, Takt 314 f. Joachims Studierexemplar

Dagegen notieren die von Leipzig unabhängigen Ausgaben von Henri Vieuxtemps, Jakob Dont und Josef Hellmesberger diesen Takt ganz oder teilweise ungebunden. ${ }^{2 \mathrm{I}}$
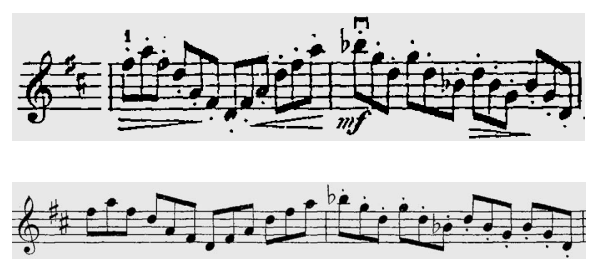

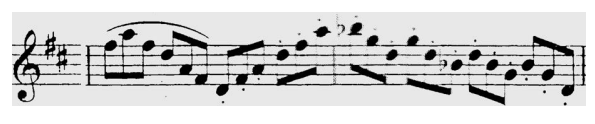

A B B ILDUNG 9 Beethoven: Violinkonzert, 1. Satz, Takt 314 f.: Vieuxtemps 1869 (links oben), Dont 1880 (rechts oben), Hellmesberger 1902 (links unten)

Joachims Ausgabe von 1905 übernimmt - wie Davids - den ganztaktigen Bindebogen. Aus dieser Tatsache allein lässt sich nicht erkennen, ob Joachim diese Version von David übernommen hat, der ja in Leipzig Joachims »Ratgeber «22 war und das Violinkonzert bereits I835 in Dorpat gespielt hatte, oder ob er einfach den I905 anerkannten und von

Ludwig van Beethoven: Concerto pour le Violon [Gebrauchsexemplar mit Eintragungen von Ferdinand David circa 1852], Wien: Tobias Haslinger [1827], British Library GB-Lbl, Tyson P.M.46. (I-3); Ludwig van Beethoven: Violinkonzert op.6r. Zum Gebrauch beim Conservatorium der Musik in Leipzig genau bezeichnet, hg. von Ferdinand David, Leipzig: Breitkopf \& Härtel [1865].

21 Ludwig van Beethoven: Violinkompositionen. Op.6r. Grosses Concert mit Piano Begleitung v. R. Volkmann, hg. von Henri Vieuxtemps, Leipzig: Schuberth [I869]; Ludwig van Beethoven: Violinkonzert op. 6r, hg. von Jacob Dont, Berlin: Schlesinger'sche Buch und Musikalienhandlung [I88o]; Ludwig van Beethoven: Concert op. 6I, hg. von Josef Hellmesberger, Leipzig: Cranz [I9O2].

22 Vgl. Andreas Moser: Joseph Joachim. Ein Lebensbild, neue, umgearb. und erw. Ausg., Bd. r, Berlin I908, S. 49-53. 
ihm so akzeptierten >Originaltext übernahm. Überraschend ist vielmehr, dass Heinrich Dessauer, der ja Joachims Auffassung wiederzugeben vorgibt, an dieser Stelle sowohl von David und den Erstausgaben als auch von Vieuxtemps und Dont abweicht und eine dritte Version im Staccato angibt: ${ }^{23}$
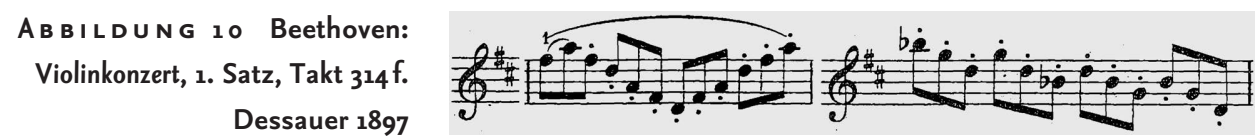

Zunächst scheint dies Dessauers Nähe zu Joachim eher in Frage zu stellen - ist seine Ausgabe demnach weiter entfernt von Joachim als zunächst angenommen? Interessanterweise finden sich ähnliche oder sogar identische Versionen dieses Taktes in weiteren Ausgaben von Joachim-Schülern, so bei Waldemar Meyer, Leopold Auer und Jenö Hubay: ${ }^{24}$
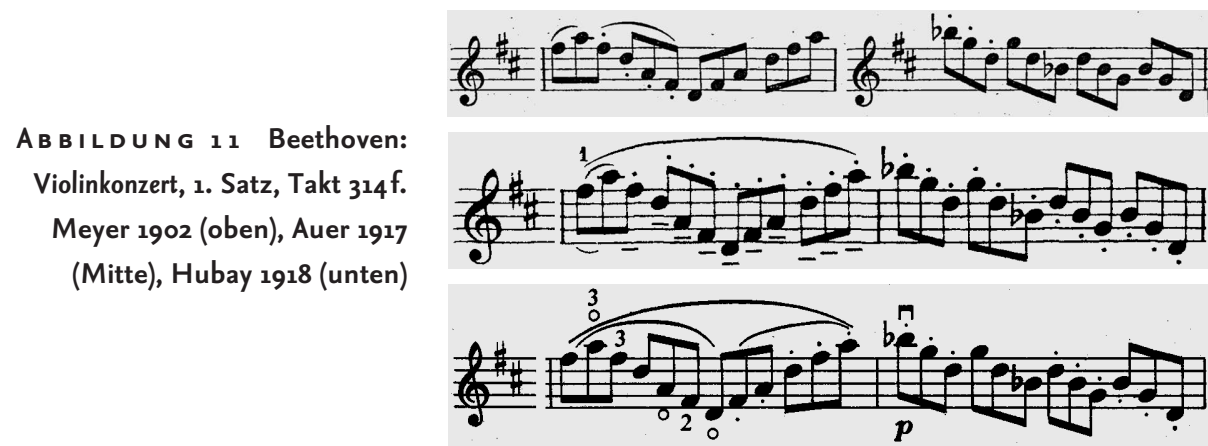

Es scheint demnach durchaus Indizien zu geben, dass Joachim diesen Takt in früheren Zeiten möglicherweise nicht gebunden, sondern - wie bei Dessauer zu finden - tatsächlich im Staccato gespielt hat. Ob dies auf eine frühere Wiener Spieltradition zurückgehen könnte, sei an dieser Stelle zunächst einmal offengelassen. Interessant und auf den ersten Blick etwas rätselhaft ist aber die Tatsache, dass diese Staccato-Version erstmals ausgerechnet in einer Ausgabe des David-Schülers August Wilhelmj von circa I883 auftaucht: ${ }^{25}$
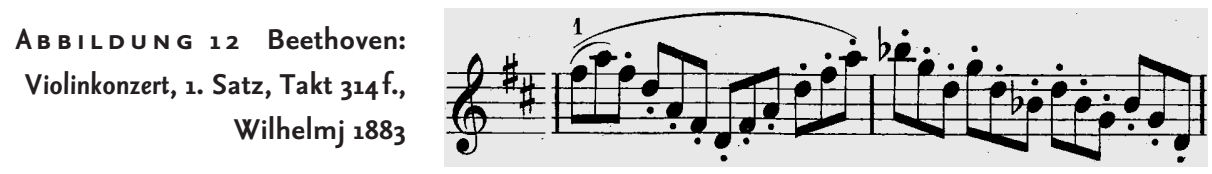

Ludwig van Beethoven: Violinkonzert op. 6r. Mit Bezeichnung und Winken unter besonderer Berücksichtigung der Auffassung von JosefJoachim für den Vortrag versehen, hg. von Heinrich Dessauer, Mainz: Schott $[1897]$.

24 Ludwig van Beethoven: Konzert für Violine mit Begleitung des Orchesters. Op.6r. Neue Ausgabe für Violine und Pianoforte, hg. von Waldemar Meyer und Robert Schwalm, Leipzig: Steingräber [1902]; Ludwig van Beethoven: Violin Concerto, Opus 6r, hg. von Leopold Auer, New York: Fischer I9I7; Ludwig van Beethoven: Violin Concerto, hg. von Jenö Hubay, Budapest: Rozsnyai rgr8.

25 Ludwig van Beethoven: Violin-Concert op. 6I, hg. von August Wilhelmj, Leipzig: C. F. Peters, PN 6718, [circa I883]. 
Rätselhaft deshalb, weil Wilhelmj zwar einerseits der Lieblings- und Vorzeigeschüler Ferdinand Davids in Leipzig war, andererseits aber Joseph Joachim eine mindestens seit I872 belegbare tiefe Abneigung gegenüber Wilhelmj hatte. ${ }^{26} \mathrm{Zu}$ erwarten gewesen wäre vielmehr eine klare Abhängigkeit von der Einrichtung seines Lehrers David. Es dürfte auszuschließen sein, dass Wilhelmj eine Wiener Spieltradition aus anderer Quelle bekannt war.

Andererseits ist ein früherer, zunächst offenbar positiver Kontakt Joachims mit Wilhelmj belegbar. Joachim erkundigt sich im Herbst I864 in zwei Briefen an Ferdinand David nach Wilhelmj ${ }^{27}$ und schreibt I867 an Bernhard Cracroft:

"You tell me you have been practising a little with Wilhelmj, and you say, , with my pupik. I wish he were; but I have even only heard him once in Leipsic, when his master David made him play to me, to show me his wonderfull [sic] ability for everything connected with the mecanisme [sic] of violin playing. I long to meet him again and to see how his talent has grown. His tone was superb than $[\mathrm{sic}] . \ll^{28}$

Hieraus scheint auch hervorzugehen, dass Wilhelmj zumindest gegenüber Cracroft den Eindruck vermittelt hatte, er sei ein Schüler Joachims, obwohl Joachim dies stark einschränkte. Ferdinand David hatte Wilhelmj vermutlich im März I864 Joachim vorgestellt, als letzterer für mehrere Konzerte, unter anderem mit dem Violinkonzert von Beethoven, in Leipzig zu Gast war. ${ }^{29}$ Es ist denkbar, dass Wilhelmj die Gelegenheit genutzt hat, mit Joachim das Violinkonzert von Beethoven durchzunehmen, zumindest aber dürfte Wilhelmj bei dieser Gelegenheit Joachim damit gehört haben. Es ist durchaus naheliegend, dass Wilhelmj sich an Joachims Auffassung orientierte; durch Briefe Wilhelmjs ist auch belegt, dass er sich immer wieder an Joachim maß:

»Heute Abend habe ich Joachim gehört und sehe nun erst ein, daß ich einmal der größte Geiger werden muß, außerdem danke ich dir noch tausend Mal für die wundervolle Geige, die mindestens 3 Mal stärker ist als die Joachim's. Er selbst meint, ich müßte der größte Geiger werden, der je gelebt, er habe noch nie einen solchen Geiger gehört.«०

Zumindest dürfte Wilhelmj Joachims Aufführung des Konzerts im Gewandhaus beigewohnt haben, und so möglicherweise Details einer Joachim'schen Auffassung übernommen haben. Umgekehrt ist es mehr als unwahrscheinlich, dass Joachim etwa Details seiner eigenen Einrichtung aus Wilhelmjs Ausgabe übernommen und dann an seine

Vgl. Brief Joachims an seine Frau vom I9. November I872, in: Briefe von und an Joseph Joachim, hg. von Johannes Joachim und Andreas Moser, 3 Bde., Berlin I9II-I9I3, Bd. 3, S. 95 f. sowie Waldemar Meyer: Aus einem Künstlerleben, Berlin I925, S.38f.

27 Vgl. Briefe von und an Joseph Joachim, Bd. 2: Die Jahre I858-1868, S. 353 und 355.

28 Ebd., S. 428.

29 Vgl. Alfred Dörffel: Statistik der Concerte im Saale des Gewandhauses zu Leipzig, Leipzig I88I, S. 8I.

30 Undatierter Brief von August Wilhelmj an seinen Vater, offenbar von I864, Privatbesitz. 
Schüler weitergegeben hätte - zu groß war seine Abneigung gegenüber Wilhelmj, als dass er dessen Ausgabe (noch dazu im Widerspruch zu den Erstausgaben) für seine eigene Interpretation herangezogen hätte. Wahrscheinlicher ist demnach, dass Wilhelmjs Ausgabe den direkten Einfluss Joachims zeigt und die Staccato-Spielweise in Takt 3I4 auf Joachim zurückgeht.

Beethovens Autograph ${ }^{3 \mathrm{I}}$ zeigt in diesem Takt eine Eigenheit, die zumindest zu einer Hypothese anregt. Hier finden sich, wie an vielen anderen Stellen, mehrere Versionen des Violinparts, wobei unzweifelhaft ist, dass die endgültige Version der in den Erstausgaben abgedruckten entspricht. Auch der ganztaktige Bindebogen ist hier zu sehen. Die ursprüngliche Version bestand aus einer anderen Dreiklangsfigur, vor allem aber scheint diese zunächst ungebunden gewesen zu sein. Der ganztaktige Bindebogen ist in dieser Urversion offensichtlich später hinzugefügt worden, erkennbar auch an den ebenfalls vorhandenen Abstoßzeichen (Punkten beziehungsweise Strichen) über den ersten drei Noten:
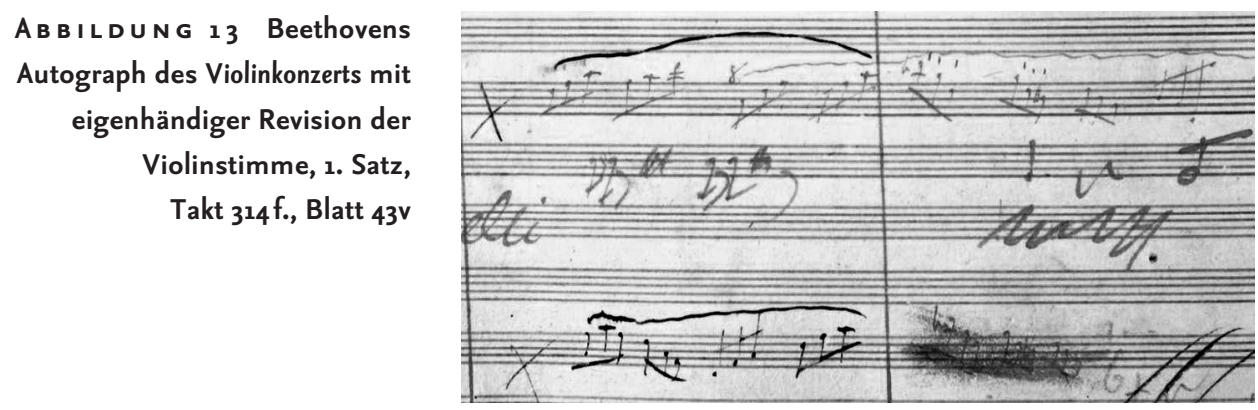

Auch wenn nicht davon auszugehen ist, dass Beethoven hier eine Ausführung im Staccato vorgesehen hatte, ist die äußerliche Ähnlichkeit in der Bezeichnung mit der unter anderem bei Wilhelmj und Dessauer abgedruckten Version offensichtlich. Sofern diese Staccato-Version auf Joachim zurückgeht - was natürlich bestenfalls eine Vermutung sein kann - lässt sich zumindest die Hypothese aufstellen, dass diese ihren Ursprung in einer früheren, von Franz Clement bei der Uraufführung gespielten Version der Violinstimme haben könnte. Es ließe sich spekulieren, dass die ursprünglich von Beethoven vorgesehene Dreiklangsfigur bis zum a'" (mit getrennten Strichen) Clement zu undankbar erschien und er sie - unter Beibehaltung der ungebundenen Strichart - in die später von Beethoven übernommene, zunächst nach unten gehende Figur geändert hatte. Beethoven hat möglicherweise in der Revision zunächst den Bindebogen oben ergänzt, dann aber die Figuration aus Clements Version übernommen, allerdings in einer gebundenen Version. So ließe sich erklären, warum in Wien eine getrennte Version des Taktes sim 




A B B IL D U N G 14 Joseph Joachims Interpretation des Violinkonzertes von Beethoven, Stemma der Abhängigkeiten und der durch Notenausgaben belegten Tradierung

Umlauf war, die sowohl von Vieuxtemps als auch von Dont und Hellmesberger - entgegen der Druckausgaben - beibehalten wurde.

Stellt man Joseph Joachims Auffassung des Violinkonzerts - als maßgebliche Werkinterpretation des I9. Jahrhunderts - in den Mittelpunkt der Untersuchung, so ergibt das resultierende Stemma der Ausgaben durch die textkritische Untersuchung bereits eine differenzierte Quellenlage, die in einer anschließenden inhaltlichen Analyse ge- 
nauere Hypothesen zu Joachims früherer Spielweise ermöglicht. Die inhaltliche Untersuchung kann nun die einzelnen Ausgaben gezielt heranziehen und nach Details suchen, in denen diese sich von Joachims Ausgabe von 1905 unterscheiden. So zeigt sich, dass die Ausgaben von Heinrich Dessauer und Waldemar Meyer, die beide überwiegend unabhängig von Ferdinand David zu sein scheinen, sich in einigen Details überraschend ähnlich sind, gleichzeitig aber von anderen Editionen abweichen, bei denen eine größere Abhängigkeit von Davids zweifellos einflussreicher Ausgabe erkennbar ist. Ergänzt man das resultierende Stemma mit der Chronologie der direkten Einflüsse und der Konzerthistorie, so ergibt sich ein differenziertes Bezugsbild, das direkte und indirekte Tradierungen erkennen lässt (Abbildung I4).

Die aus der Editionsphilologie übernommene textkritische Herangehensweise kann in der Interpretationsforschung genutzt werden, um Abhängigkeiten von Interpreten beziehungsweise von Interpretationen anhand von annotierten Notenausgaben zu untersuchen. Die daraus resultierenden Stemmata erlauben in vielen Fällen eine differenzierte Aufschlüsselung von Tradierungen, die durch die rein inhaltliche Untersuchung möglicherweise unsichtbar geblieben wären. Lokale Ausprägungen, regionale Querverbindungen und persönliche Beziehungen werden in vielen Fällen bereits sichtbar, noch bevor die biografischen Details berücksichtigt werden. Abhängigkeiten und Abgrenzungen lassen sich so mit Hilfe bewährter Methodik erkennen und benennen, was letztlich eine objektivere Bewertung der Zusammenhänge erlaubt. 


\section{Inhalt}

Vorwort 8

INTERPRETATION - BEGRIFF, METHODE, PRAXIS

Laure Spaltenstein Interpretation als treue Übersetzung. Zur Frühgeschichte eines vieldeutigen Begriffs I5

Kai Köpp Von der Quelle zur Methode. Zum Entwurf einer historischen Interpretationsforschung $\quad 28$

Manuel Bärtsch >Interpretation‘. Beethovens Sonate A-Dur op. IOI in der Sicht von Eugen d'Albert und Frederic Lamond

Sebastian Bausch Klavierrollen als Interpretationsdokumente. Ein Erfahrungsbericht als Leitfaden für Einsteiger $\quad 7 \mathrm{I}$

Camilla Köhnken Beethoven-Auslegung zwischen Liszts »Deklamationsstil« und Bülows »Vivisektionsversuchen«. Auf den Spuren Liszt'scher Interpretationsideale in Hans von Bülows instruktiver Edition der Klaviersonaten Beethovens $\quad 92$

Neal Peres Da Costa Carl Reinecke's Performance of his Arrangement of the Second Movement from Mozart's Piano Concerto K. 488. Some Thoughts on Style and the Hidden Messages in Musical Notation

II4

Carolina Estrada Bascuñana Enrique Granados's Performance Style.

Visualising the Audible Evidence I5O

Lukas Näf Tempogestaltung in Weberns Sinfonie op. 2I I80

INTERPRETATION - AUFFÜHRUNGSGESCHICHTE

Christoph Moor "Ein so erklärtes Lieblingsstück der hiesigen Kunstfreunde«. Die Rezeptionsgeschichte der Jupiter-Sinfonie in Beethovens Wien

Luisa Klaus Objektive Bruckner-Interpretation? Zur Aufführung der Trio-Entwürfe für die Neunte Sinfonie I940 205

Chris Walton Von innen und von außen. Beethovens Neunte Sinfonie und die $>$ Wagner'sche< Dirigiertradition $\quad 2 \mathrm{I} 8$

Lena-Lisa Wüstendörfer Streit um Fidelio. Gustav Mahler und Felix Weingartner im Disput um Werktreue $\quad 238$

INTERMEZZO

Robert Levin Turning Point to Musical Modernity. Beethoven as Executor of the Legacy of C. P. E. Bach. Concert Lecture 249

INTERPRETATION - INSTRUMENTE, ANALYSE, EDITION

Martin Skamletz «Man hat diese Erweiterung des Tonumfanges seit ein paar Jahren an den Tasteninstrumenten sehr weit getrieben.« Der Umgang mit Grenzen beim späten Mozart und beim frühen Beethoven $\quad 263$ 
Stephan Zirwes Analyse und Interpretation. Adolph Bernhard Marx' Beethoven-Analysen 29I

Michael Ladenburger Was können wir aus Originalhandschriften von Beethoven für eine angemessene Interpretation lernen? 30I

Federica Rovelli Die Skizzenbuch-Ausgaben und ihre mögliche digitale Zukunft 3 I7

Johannes Gebauer Interpretationspraktische Stemmatik. Philologische Methoden in der Interpretationsforschung am Beispiel annotierter Notenausgaben von Rodes 24 Capricen und Beethovens Violinkonzert

John Rink Chopin Copying Chopin 349

Tomasz Herbut Alexander Goldenweiser und Beethovens Sonate op. IIo- eine Spurensuche 366

INTERPRETATION - KREATIVE ANEIGNUNG

Thomas Gartmann Beethoven als sein eigener Interpret. Gedanken zur Bearbeitung der Klaviersonate op. I4/ז für Streichquartett

Ivo Haag Die Sinfonien von Johannes Brahms - (auch) Klaviermusik?

Michael Lehner Das Orchester auf dem Klavier. Welte-Klavierrollen von Gustav Mahler und Richard Strauss als interpretationsanalytische Quellen

Roger Allen “That Is What Music Really Is". Richard Wagner's Reception of Beethoven's Piano Sonata in A Major Op. IOI $43 \mathrm{I}$

Daniel Allenbach Eine heroische< Neunte? Dmitri Schostakowitschs Neunte Sinfonie im Vergleich mit Ludwig van Beethoenvs Sinfonien Nr.3 und 9 44I

Simeon Thompson Beethoven und der Zweite Weltkrieg in der künstlerischen Reflexion der Nachkriegszeit. Stanley Kubricks A Clockwork Orange und Rolf Liebermanns Leonore 40/45 456

Michelle Ziegler Rettungsversuch im Jubiläumsjahr. Mauricio Kagels Aufarbeitung der Beethoven-Rezeption in der Ludwig van-Werkgruppe (I970) 465

Leo Dick Über den späten Beethoven zur >Postidentität،. Die Suche nach liminalen Räumen im gegenwärtigen Musiktheater am Beispiel von Matthias Rebstocks Berliner Produktion Büro für postidentisches Leben $\quad 476$

Elizabeth Waterhouse Choreographic Re-mix. William Forsythe's Trio (I996) and Beethoven's String Quartet No. I5 in a Minor Op. I32 487

László Stachó "Gradus ad Parnassum".

The Purgatory of Instrumental Technique

Namen-, Werk- und Ortsregister 522

Die Autorinnen und Autoren der Beiträge 


\section{Rund um BeEthoven \\ Interpretationsforschung heute • \\ Herausgegeben von Thomas}

Gartmann und Daniel Allenbach 


\section{MUSIKFORSCHUNG DER Hochschule der KÜnste Bern Herausgegeben von Martin Skamletz und Thomas Gartmann Band 14}


0 Dieses Buch ist in gedruckter Form im Dezember 2019 in erster Auflage in der Edition Argus in Schliengen/Markgräflerland erschienen. Gestaltet und gesetzt wurde es im Verlag aus der Seria und der SeriaSans, die von Martin Majoor im Jahre 2000 gezeichnet wurden. Gedruckt wurde es auf Eos, einem holzfreien, säurefreien, chlorfreien und alterungsbeständigen Werkdruckpapier der Papierfabrik Salzer im niederösterreichischen Sankt Pölten. Das Vorsatzpapier Caribic cherry wurde von Igepa in Hamburg geliefert. Rives Tradition, ein Recyclingpapier mit leichter Filznarbung, das für den Bezug des Umschlags verwendet wurde, stellt die Papierfabrik Arjo Wiggins in Issy-les-Moulineaux bei Paris her. Das Kapitalband mit rot-schwarzer Raupe lieferte die Firma Dr. Günther Kast aus Sonthofen im Oberallgäu, die auf technische Gewebe und Spezialfasererzeugnisse spezialisiert ist. Gedruckt und gebunden wurde das Buch von der Firma Bookstation im bayerischen Anzing. Im Internet finden Sie Informationen über das gesamte Verlagsprogramm unter www.editionargus.de, zum Institut Interpretation der Hochschule der Künste Bern unter www.hkb.bfh.ch/interpretation und www.hkb-interpretation.ch. Die Deutsche Nationalbibliothek verzeichnet diese Publikation in der Deutschen Nationalbibliografie; detaillierte bibliografische Daten sind im Internet über www.dnb.de abrufbar. (c) der zeitgleich erschienenen digitalen Version: die Autorinnen und Autoren, 20I9. Dieses Werk ist lizenziert unter einer Creative Commons Namensnennung-Nicht kommerziell 4.0 International Lizenz (CC BY-NC 4.o). DoI: https://doi.org/I0.26045/kp64-6I78 ISBN 978-3-93I264-94-9 